\title{
Estimates of energy expenditure using the RT3 accelerometer in patients with systemic lupus erythematosus
}

\author{
Tim K. Tso ${ }^{1 *}$, Wen-Nan Huang ${ }^{2}$, Chen-Kang Chang ${ }^{3}$ \\ ${ }^{1}$ Department of Food Science, National Chiayi University, Chia-Yi, Taiwan, China; \\ *Corresponding Author: timtso@mail.ncyu.edu.tw \\ ${ }^{2}$ Department of Allergy, Immunology and Rheumatology, Taichung Veterans General Hospital, Taichung, Taiwan, China \\ ${ }^{3}$ Sport Science Research Center, Taiwan Sport University, Taichung, Taiwan, China
}

Received 16 November 2009; revised 15 January 2010; accepted 1 February 2010.

\section{ABSTRACT}

This study aimed to characterize energy expenditure patterns using the tri-axial accelerometer and to identify the association of energy expenditure with clinical parameters in patients with systemic lupus erythematosus (SLE). Estimates of energy expenditures represented by total activity calorie (TA), physical activity calorie (PA), total activity calorie per body weight (TABW), and physical activity calorie per body weight (PABW) of 49 female SLE patients were assessed using the RT3 tri-axial accelerometer (StayHealthy, Monrovia, CA) in a seven-day period. SLE patients in the highest body mass index (BMI) tertile showed significantly lower values of TABW compared to those in the lowest tertile, while SLE patients in the lowest TABW tertile showed significantly higher body weight, waist circumference, BMI, SLE disease activity index (SLEDAI), dosage of prednisone, and blood pressure. There was a high prevalence of metabolic syndrome and SLE patients with metabolic syndrome showed significantly lower TABW. In addition, both TABW and PABW significantly but negatively correlated with SLEDAI. In conclusion, the RT3 accelerometer is suitable for evaluating total and physical activity-related energy expenditure in patients with SLE. TABW measured by the tri-axial accelerometer is inversely related with body weight status and disease activity in SLE patients. This suggests that estimates of energy expenditure by the tri-axial accelerometer may be applied in the management of SLE.

Keywords: Accelerometer; Body mass index;
Energy Expenditure; Systemic Lupus Erythematosus

\section{INTRODUCTION}

Systemic lupus erythematosus (SLE) is a chronic autoimmune disease characterized not only by the activation of $\mathrm{T}$ and polyclonal $\mathrm{B}$ cells but also by a wide variety of immunologic abnormalities [1]. Previous studies demonstrate that SLE patients present with limitations in exercise capacity and reduced quality of life due to various clinical complaints [2]. The exercise intolerance of SLE patients is associated with a reduced aerobic capacity of peripheral muscles [3], such that SLE patients are less aerobically fit, with reduced exercise capacity, reduced muscle strength, more fatigue, and greater disability compared to sedentary controls [4].

The incidence of SLE is higher in females than in males. There is strong evidence on the relationship between physical activity and the primary prevention of chronic health problems in women [5]. Estimates of energy expenditure provided a better indicator of physical activity. However, methods to assess energy expenditure have strengths and limitations [6].

The RT3 accelerometer, an integrated tri-axial accelerometer giving output measures in medio-lateral, anterior-posterior, and vertical dimensions, is a valid tool for assessing physical activity [7-9]. Although its reliability, validation, and application in measuring physical activeity in different population have been reported [10-15], the relationship between disease activity and energy expenditure measured by the RT3 accelerometer in SLE patients has not been previously established.

This study aimed to characterize energy expenditure patterns using the RT3 accelerometer and identify any relationship between energy expenditure and clinical parameters in SLE patients. 


\section{MATERIALS AND METHODS}

Forty-nine (49) Chinese female SLE patients aged 20-50 years who fulfilled the American College of Rheumatology criteria [16] and had no contraindications to physical activity were randomly selected from the outpatient clinics of Taichung Veterans General Hospital (Taichung, Taiwan). The hospital's ethical committee approved the study and all SLE patients provided written informed consent.

Energy expenditure was assessed using the RT3 Triaxial Activity Measurement and Recording System (StayHealthy, Monrovia, CA, USA). The RT3 was a relatively small device (the size of a pager) and used an integrated tri-axial accelerometer. These were worn by the SLE patients in nylon pouches secured to a belt at the waistline above each hip during the seven-day period. The patients' profiles, including age, height, and weight, were entered into the RT3 accelerometer and the acceleration was measured periodically. Information obtained was then converted into energy expenditure estimates that included total activity calorie (TA), physical activity calorie (PA), total activity calorie per body weight (TABW), and physical activity calorie per body weight (PABW).

Anti-dsDNA was measured by enzyme-linked immunosorbent assay using a Quanta Lite $^{\mathrm{TM}}$ dsDNA Kit (INOVA Diagnostics Inc., San Diego, CA, USA). Quantitative determinations of serum $\mathrm{C} 3$ and $\mathrm{C} 4$ were conducted using $\mathrm{N}$ Antisera to Human Complement Factor reagents with the Behring Nephelometers (Dade Behring, Inc., Newark, DE, USA). Disease activity was determined using the SLE Disease Activity Index (SLEDAI) [17], while enzymatic methods were used to determine circulating concentrations of total cholesterol (Beckman TC Reagent) and triglyceride (TG) (Beckman TG Reagent). Magnesium-dextran sulfate precipitation reagent was used to separate high-density lipoprotein-cholesterol (HDL-C), which was then assessed enzymatically. Low-density lipoprotein-cholesterol (LDL-C) was determined by the Friedewald equation [18].

Metabolic syndrome was based on the National Cholesterol Education Program (NCEP/ATP III) definition [19]. SLE patients were defined as having metabolic syndrome in the presence of three or more of the following criteria: waist circumference $>88 \mathrm{~cm}$; systolic blood pressure $>130 \mathrm{~mm} \mathrm{Hg}$ or diastolic blood pressure $>85 \mathrm{mmHg}$; HDL-C $<50 \mathrm{mg} / \mathrm{dL} ; \mathrm{TG}>150 \mathrm{mg} / \mathrm{dL}$; and fasting blood glucose $>110 \mathrm{mg} / \mathrm{dL}$.

Statistical analyses were performed using the Statistical Package of Social Sciences (SPSS) 10.0 for Windows (SPSS Inc., Chicago, IL, USA). Tested variables for comparison of means were expressed as mean \pm standard deviation and 95\% confidence interval. The distribution of tested variables was examined graphically for normality. Kruskal-Wallis test and Mann-Whitney U test were used to examine the mean differences of energy expenditure between the SLE patients stratified by BMI and metabolic syndrome. Analysis of variance (ANOVA) was also used to examine the mean differences of test parameters among SLE patients stratified by tertiles of TABW as measured by the RT3 accelerometer. In addition, Pearson's or Spearman correlation analysis was used accordingly to examine the relationships between estimates of energy expenditure and test variables. A $p$ value $<0.05$ was considered significant for all statistical analyses in this study.

\section{RESULTS}

All of the patients were non-smokers, performed regular daily activities, and did not attend any supervised exercise training program during the experiment period. Average estimates of energy expenditures measured by the RT3 accelerometer were $1730 \pm 218 \mathrm{Kcal} /$ day TA, $284 \pm$ $108 \mathrm{Kcal} /$ day PA, $26 \pm 3 \mathrm{Kcal} / \mathrm{kg} /$ day TABW, and $4 \pm 1$ $\mathrm{Kcal} / \mathrm{kg}$ /day PABW. Thirty-six patients $(74 \%)$ had a normal BMI $\left(<25 \mathrm{~kg} / \mathrm{m}^{2}\right)$, nine $(18 \%)$ were overweight $\left(25-29.9 \mathrm{~kg} / \mathrm{m}^{2}\right)$, and four $(8 \%)$ were obese $\left(>30 \mathrm{~kg} / \mathrm{m}^{2}\right)$. Compared to patients with normal BMI, overweight or obese patients had higher SLEDAI (mean: $3.38 \pm 2.50$, $\mathrm{n}$ $=13$ vs. $1.28 \pm 1.80, \mathrm{n}=36, p=0.013)$ and lower TABW (mean: $23.42 \pm 1.92 \mathrm{Kcal} / \mathrm{kg} /$ day, $\mathrm{n}=13$ vs. $27.25 \pm 2.60 \mathrm{Kcal} / \mathrm{kg} / \mathrm{day}, \mathrm{n}=36, p<0.001)$. The median SLEDAI value in all patients was 2 and only $31 \%$ of patients had SLEDAI scores higher than 3, indicating that most of the patients had stable or moderately active disease status [20]. However, SLEDAI significantly correlated with body weight $(\mathrm{r}=0.434, p=0.002)$ and BMI $(\mathrm{r}=0.379, p=0.007)$. Both TABW $(\mathrm{r}=-0.303, p=$ $0.034)$ and PABW $(\mathrm{r}=-0.301, p=0.035)$ negatively correlated with SLEDAI.

Estimates of energy expenditure measured by the RT3 accelerometer in SLE patients stratified by BMI were shown in Table 1. SLE patients in the highest BMI tertile showed significantly higher TA and PA, and signifycantly lower TABW compared to those in the lowest tertile. In addition, there was a significant negative correlation between BMI and TABW $(\mathrm{r}=-0.668, p<$ 0.001 ).

Estimates of energy expenditure in SLE patients with and without metabolic syndrome were shown in Table 2. According to the NCEP/ATP III definition, 21 patients (43\%) met the criteria for metabolic syndrome. They had significantly higher TA and PA, and significantly lower TABW. 
Table 1. Estimates of energy expenditure by RT3 accelerometer in SLE patients stratified by body mass index $(\mathrm{n}=49)^{\mathrm{a}}$.

\begin{tabular}{lcccc}
\hline & \multicolumn{3}{c}{ Body mass index $\left(\mathrm{kg} / \mathrm{m}^{2}\right)$ tertiles } & Overall P value \\
\cline { 2 - 4 } & $<20.5 \mathrm{~kg} / \mathrm{m}^{2}$ & $20.5-23.6 \mathrm{~kg} / \mathrm{m}^{2}$ & $>23.6 \mathrm{~kg} / \mathrm{m}^{2}$ & \\
\hline $\mathrm{TA}(\mathrm{Kcal} / \mathrm{day})$ & $1611 \pm 118(1549-1674)$ & $1696 \pm 263(1561-1831)$ & $1886 \pm 147(1808-1964)$ & $<0.001^{*}$ \\
$\mathrm{TABW}(\mathrm{Kcal} / \mathrm{kg} /$ day $)$ & $28.11 \pm 2.10(26.99-29.22)$ & $26.50 \pm 2.97(24.97-28.02)$ & $24.09 \pm 2.35(22.84-25.34)$ & $<0.001^{*}$ \\
$\mathrm{PA}(\mathrm{Kcal} / \mathrm{day})$ & $244 \pm 79(202-287)$ & $263 \pm 98(213-313)$ & $347 \pm 119(284-411)$ & $0.027^{*}$ \\
PABW (Kcal/kg/day) & $4.22 \pm 1.19(3.58-4.85)$ & $4.10 \pm 1.30(3.43-4.77)$ & $4.56 \pm 1.54(3.74-5.38)$ & 0.694 \\
\hline
\end{tabular}

Abbreviations: TA, Total activity calorie; TABW, Total activity calorie per body weight; PA, Physical activity calorie; PABW, Physical activity calorie per body weight

${ }^{\mathrm{a}}$ Values are expressed as mean \pm standard deviation and $95 \%$ confidence interval.

${ }^{\mathrm{b}}$ Statistical significance $\left({ }^{*} p<0.05\right)$ as determined by the Kruskal-Wallis test.

Table 2. Estimates of energy expenditure by RT3 accelerometer in SLE patients with and without metabolic syndrome ${ }^{\mathrm{a}}$.

\begin{tabular}{lccc}
\hline & $\begin{array}{c}\text { SLE patients without } \\
\text { metabolic syndrome }(\mathrm{n}=28)\end{array}$ & $\begin{array}{c}\text { SLE patients with } \\
\text { metabolic syndrome }(\mathrm{n}=21)\end{array}$ & P value $^{\mathrm{b}}$ \\
\hline TA (Kcal/day) & $1668 \pm 149(1602-1734)$ & $1894 \pm 267(1772-2016)$ & $0.001^{*}$ \\
TABW (Kcal/kg/day) & $31.56 \pm 2.70(30.36-32.76)$ & $29.78 \pm 4.25(27.85-31.72)$ & $0.009^{*}$ \\
PA (Kcal/day) & $264 \pm 101(220-309)$ & $390 \pm 226(287-493)$ & $0.039^{*}$ \\
PABW (Kcal/kg/day) & $4.97 \pm 1.79(4.18-5.77)$ & $6.06 \pm 3.53(4.45-7.67)$ & 0.409 \\
\hline
\end{tabular}

Abbreviations: TA, Total activity calorie; TABW, Total activity calorie per body weight; PA, Physical activity calorie; PABW, Physical activity calorie per body weight

${ }^{a}$ Values are expressed as mean \pm standard deviation and $95 \%$ confidence interval.

${ }^{\mathrm{b}}$ Statistical significance $\left({ }^{*} p<0.05\right)$ was determined by Mann-Whitney U test.

The mean differences of anthropometric measurements, disease activity-related variables, and lipid profile between patients stratified into tertiles based on TABW were shown in Table 3. SLE patients in the lowest TABW tertile showed higher body weight, waist circumference, BMI, SLEDAI, dosage of prednisone, and blood pressure. Patients in the higher TABW tertile tended to have reduced concentrations of total cholesterol, TG and LDL-C but the differences were not statistically significant.

\section{DISCUSSION}

This study evaluated estimates of energy expenditure by using the RT3 accelerometer in 49 female SLE patients. The major finding was that estimates of energy expenditure represented as daily TABW and PABW were negatively associated with disease activity.

There is a number of assessments available for evaluating physical activity, such as self-administered questionnaires of physical activity, pedometers, accelerometers, and supervised cardio-vascular training programs $[11,21,22]$. However, each method has its own strengths and limitations. The RT3 accelerometer is used to assess physical activity in children [10,11], adolescents [11], overweight adults [12], pregnant women [13], individuals with multiple sclerosis [14], and older adults with coronary heart disease [15]. Klassen et al. report that the RT3 accelerometer can detect a significant difference between moderately active and active individuals with multiple sclerosis [14]. Chu et al. have demonstrated that RT3 movement counts increases in a linear manner with scaled oxygen uptake from stationary to vigorous intensity movement in children [10]. In the present study, the RT3 accelerometer is used to assess estimates of energy expenditure in SLE patients and is proven to be userfriendly and an acceptable measure of free-living physiccal activity in such population. The RT3 accelerometer appears to distinguish estimates of energy expenditure in SLE patients with varying clinical characteristics.

Recent studies demonstrate the beneficial effects of exercise in SLE patients in terms of improving fatigue, physical function, aerobic fitness, and quality of life [22-25]. Aerobic exercises performed on a treadmill do not aggravate disease activity at any time during an exercise period and may provide some benefits on fatigue 
Table 3. Characteristics of SLE patients in the respective tertiles of total activity calorie per body weight $(n=49)^{\mathrm{a}}$.

\begin{tabular}{|c|c|c|c|c|}
\hline & \multicolumn{3}{|c|}{ Total activity calorie per body weight (Kcal/kg/day) tertiles } & \multirow{2}{*}{ Overall $\mathrm{P}$ value $\mathrm{b}^{\mathrm{b}}$} \\
\hline & $<24.91(\mathrm{Kcal} / \mathrm{kg} /$ day $)$ & $24.91-27.17$ (Kcal/kg/day) & $>27.17(\mathrm{Kcal} / \mathrm{kg} / \mathrm{day})$ & \\
\hline Weight (kg) & $66.87 \pm 9.95(61.56-72.17)$ & $55.74 \pm 6.10(52.49-58.99)$ & $50.15 \pm 6.12(47.00-53.29)$ & $<0.001^{*}$ \\
\hline $\operatorname{BMI}\left(\mathrm{kg} / \mathrm{m}^{2}\right)$ & $26.96 \pm 4.69(24.45-29.46)$ & $22.03 \pm 1.93(20.99-23.06)$ & $20.62 \pm 2.30(19.43-21.80)$ & $<0.001^{*}$ \\
\hline Waist circumference $(\mathrm{cm})$ & $88.39 \pm 12.65(81.09-95.69)$ & $78.70 \pm 8.77(73.85-83.55)$ & $74.89 \pm 5.49(71.72-78.06)$ & $0.002 *$ \\
\hline Anti-dsDNA (IU/ml) & $129.14 \pm 181.57(32.38-225.89)$ & $115.49 \pm 133.08(44.57-186.40)$ & $151.50 \pm 195.41(51.03-251.97)$ & 0.833 \\
\hline $\mathrm{C} 3(\mathrm{mg} / \mathrm{dl})$ & $84.58 \pm 22.73(72.46-96.69)$ & $93.93 \pm 20.71(82.89-104.96)$ & $89.34 \pm 16.77(80.72-97.97)$ & 0.429 \\
\hline $\mathrm{C} 4(\mathrm{mg} / \mathrm{dl})$ & $16.09 \pm 9.19(11.19-20.99)$ & $16.20 \pm 9.61(11.08-21.32)$ & $18.11 \pm 9.08(13.44-22.78)$ & 0.781 \\
\hline SLEDAI & $3.38 \pm 2.28(2.16-4.59)$ & $1.00 \pm 1.79(0.05-1.95)$ & $1.18 \pm 1.74(0.28-2.07)$ & $0.002 *$ \\
\hline Prednisone (mg/day) & $12.03 \pm 5.18(9.27-14.79)$ & $6.88 \pm 4.13(4.67-9.08)$ & $7.79 \pm 6.67(4.37-11.22)$ & $0.023^{*}$ \\
\hline $\mathrm{TC}(\mathrm{mmol} / \mathrm{l})$ & $5.32 \pm 1.18(4.70-5.95)$ & $5.21 \pm 1.01(4.67-5.75)$ & $4.65 \pm 0.86(4.21-5.09)$ & 0.139 \\
\hline TG (mmol/l) & $1.48 \pm 0.77(1.07-1.89)$ & $0.99 \pm 0.34(0.81-1.17)$ & $1.20 \pm 0.50(0.94-1.46)$ & 0.061 \\
\hline LDL-C (mmol/l) & $2.94 \pm 1.03(2.39-3.49)$ & $2.82 \pm 0.72(2.44-3.20)$ & $2.49 \pm 0.60(2.19-2.80)$ & 0.264 \\
\hline HDL-C (mmol/l) & $1.71 \pm 0.49(1.45-1.97)$ & $1.93 \pm 0.62(1.61-2.26)$ & $1.61 \pm 0.47(1.37-1.85)$ & 0.207 \\
\hline TC/HDL-C & $3.36 \pm 0.88(2.86-3.87)$ & $2.83 \pm 0.50(2.55-3.10)$ & $3.15 \pm 0.76(2.71-3.59)$ & 0.144 \\
\hline SBP $(\mathrm{mmHg})$ & $153 \pm 29.22(136.13-169.87)$ & $126.13 \pm 28.37(110.42-141.84)$ & $121.29 \pm 14.92(112.67-129.90)$ & $0.004 *$ \\
\hline $\mathrm{DBP}(\mathrm{mmHg})$ & $91.14 \pm 19.35(79.97-102.32)$ & $78.67 \pm 16.70(69.42-87.92)$ & $74.43 \pm 12.99(66.93-81.93)$ & $0.029 *$ \\
\hline
\end{tabular}

Abbreviations: BMI, body mass index; C3, complement factor 3; C4, complement factor 4; SLEDAI, systemic lupus erythematosus disease activity index; TC, total cholesterol; TG, triglyceride; LDL-C, low-density lipoprotein-cholesterol; HDL-C, high-density lipoprotein-cholesterol; SBP, systolic blood pressure; DBP, diastolic blood pressure

${ }^{a}$ Values are expressed as mean \pm standard deviation and $95 \%$ confidence interval.

${ }^{\mathrm{b}}$ Statistical significance $\left({ }^{*} p<0.05\right)$ as determined by ANOVA test.

and physical function in SLE patients with low disease activity [25]. In a 12-week supervised cardio-vascular training program, SLE patients have shown improved exercise tolerance, aerobic capacity, oxygen pulse, fatigue, quality of life, depression, and functional capacity [22]. In a pilot study on the effect of exercise in SLE patients, both aerobic and range of motion/muscle strengthening types of exercises are safe and do not worsen SLE disease activity [23]. In the present study, SLE patients with lowest TABW tertile show higher SLEDAI and dosage of prednisone. It also identifies both TABW and PABW to be inversely correlated with SLEDAI, suggesting that improved daily energy expenditure may be beneficial to disease management in SLE.

Obesity is independently associated with impaired functional capacity and health-related quality of life in patients with SLE [26] and disease activity is predictive of deleterious increases in BMI [27]. Although only $26 \%$ of patients in this study are overweight or obese with BMI $>25 \mathrm{~kg} / \mathrm{m}^{2}$, they have higher SLEDAI and lower TABW. SLE patients in the lowest TABW tertile show the highest body weight, waist circumference, BMI, SLEDAI, dosage of prednisone, and blood pressure, as well as a positive correlation between BMI and SLEDAI $(\mathrm{r}=0.379, p=0.007)$. Taken together, the findings here suggest that less energy expenditure corresponding to increased BMI may aggravate, at least partly, the disease activity in female SLE patients. However, due to lack of self-reported information about the time record and types of activity when the RT3 accelerometer was on and off, physical activity intensity and metabolic equivalent energy expenditure cannot be completely evaluated.

Recent studies demonstrate a higher prevalence of insulin resistance and metabolic syndrome in SLE patients $[28,29]$. The high prevalence of metabolic syndrome is confirmed in the present study wherein 21 of $49(43 \%)$ SLE patients have metabolic syndrome using the NCEP/ ATP III definition. Physical activity energy expenditure can predict progression towards metabolic syndrome in middle-aged healthy Caucasians [30]. SLE patients with metabolic syndrome in the present study show signifycantly lower TABW. In addition, patients with higher 
TABW tend to have reduced concentrations of total cholesterol, TG, and LDL-cholesterol. However, the lipid profiles of most patients in this study are within normal range and not considered dyslipidemic [19], which can limit possible effect of physical activity-related energy expenditure on circulating lipid levels.

In conclusion, as increasing physical activity can be a component of lifestyle interventions designed to improve quality of life and functional capacity in SLE patients, this study demonstrates the relationship among estimates of energy expenditure measured by RT3 accelerometer, disease activity, and components of metabolic syndrome. Incorporating physical activity-related energy expenditure into the clinical management of SLE may be beneficial.

\section{ACKNOWLEDGEMENTS}

This study was supported by a research grant from the National Science Council of Taiwan (NSC 96-2413-H-415-006-MY2). The authors wish to thank the Biostatistics Task Force of Taichung Veterans General Hospital for their assistance in the statistical analysis. The authors also thank Ms Ging-Yi Lin for her technical assistance.

\section{REFERENCES}

[1] D'Cruz, D.P., Khamashta, M.A. and Hughes, G.R. (2007) Systemic lupus erythematosus. Lancet, 369(9561), 587596.

[2] Vu, T.V. and Escalante, A. (1999) A comparison of the quality of life of patients with systemic lupus erythematosus with and without end-stage renal disease. Journal of Rheumatology, 26(12), 2595-2601.

[3] Forte, S., Carlone, S., Vaccaro, F., Onorati, P., Manfredi, F., Serra, P. and Palange, P. (1999) Pulmonary gas exchange and exercise capacity in patients with systemic lupus erythematosus. Journal of Rheumatology, 26(12), 2591-2594.

[4] Tench, C., Bentley, D., Vleck, V., McCurdie, I., White, P. and D'Cruz, D. (2002) Aerobic fitness, fatigue, and physical disability in systemic lupus erythematosus. Journal of Rheumatology, 29(3), 474-481.

[5] Brown, W.J., Burton N.W. and Rowan P.J. (2007) Updating the evidence on physical activity and health in women. American Journal of Preventive Medicine, 33(5), 404-411.

[6] Lagerros Y.T. and Lagiou P. (2007) Assessment of physical activity and energy expenditure in epidemiological research of chronic diseases. European Journal of Epidemiology, 22(6), 353-362.

[7] DeVoe, D., Gotshall, R. and McArthur, T. (2003) Comparison of the RT3 research tracker and tritrac R3D accelerometers. Percept Mot Skills, 97(2), 510-518.

[8] Powell, S.M. and Rowlands, A.V. (2004) Intermonitor variability of the RT3 accelerometer during typical physical activities. Medicine and Science in Sports and Exercise, 36(2), 324-330.
[9] Rowlands, A.V., Thomas, P.W., Eston, R.G. and Topping, R. (2004) Validation of the RT3 tri-axial accelerometer for the assessment of physical activity. Medicine and Science in Sports and Exercise, 36(3), 518-524.

[10] Chu, E.Y., McManus, A.M. and Yu, C.C. (2007) Calibration of the RT3 accelerometer for ambulation and nonambulation in children. Medicine and Science in Sports and Exercise, 39(11), 2085- 2091.

[11] De Vries, S.I., Bakker, I., Hopman-Rock, M., Hirasing, R.A. and Van Mechelen, W. (2006) Clinimetric review of motion sensors in children and adolescents. Journal of Clinical Epidemiology, 59(7), 670-680.

[12] Jacobi, D., Perrin, A.E., Grosman, N., Dore, M.F., Normand, S., Oppert, J.M. and Simon, C. (2007) Physical activity-related energy expenditure with the RT3 and TriTrac accelerometers in overweight adults. Obesity, 15 (4), 950-956.

[13] DiNallo, J.M., Le, Masurier, G.C., Williams, N.I. and Downs, D.S. (2008) Walking for health in pregnancy: assessment by indirect calorimetry and accelerometry. Research Quarterly for Exercise and Sport, 79(1), 28-35.

[14] Klassen, L., Schachter, C. and Scudds, R. (2008) An exploratory study of two measures of free-living physical activity for people with multiple sclerosis. Clinical Rehabilitation, 22(3), 260-271.

[15] Orrell, A., Doherty, P., Miles, J. and Lewin, R. (2007) Development and validation of a very brief questionnaire measure of physical activity in adults with coronary heart disease. European Journal of Cardiovascular Prevention and Rehabilitation, 14(5), 615-623.

[16] Tan, E.M., Cohen, A.S., Fries, J.F., Masi, A.T., McShane, D.J., Rothfield, N.F., Schaller, J.G., Talal, N. and Winchester, R.J. (1982) The 1982 revised criteria for the classification of systemic lupus erythematosus. Arthritis and Rheumatism, 25(11), 1271-1277.

[17] Bombardier, C., Gladman, D.D., Urowitz, M.B., Caron, D., Chang, C.H. and the Committee on Prognosis Studies in SLE (1992) Derivation of the SLEDAI: A disease activity index for lupus patients. Arthritis and Rheumatism, 35(6), 630-640.

[18] Friedewald, W.T., Levy, R.I. and Fredrickson, D.S. (1972) Estimation of the concentration of low-density lipoprotein cholesterol in plasma without use of the preparative ultracentrifuge. Clinical Chemistry, 18(6), 499-502.

[19] Expert Panel on Detection, Evaluation, and Treatment of High Blood Cholesterol in Adults (2001) Executive Summary of the Third Report of the National Cholesterol Education Program (NCEP) Expert Panel on Detection, Evaluation, And Treatment of High Blood Cholesterol In Adults (Adult Treatment Panel III). Journal of the American Medical Association, 285, 2486-2497.

[20] Chang, D.M., Lan, J.L., Lin, H.Y. and Luo, S.F. (2002) Dehydroepiandrosterone treatment of women with mildto-moderate systemic lupus erythematosus. Arthritis and Rheumatism, 46(11), 2924-2927.

[21] Faulkner, G., Cohn, T. and Remington, G. (2006) Validation of a physical activity assessment tool for individuals with schizophrenia. Schizophrenia Research, 82(3), 225231.

[22] De Carvalho, M.R.P., Sato, E.I., Tebexreni, A.S., Heidecher, R.T.C., Schenkman, S. and Barros Neto, T.L. (2005) Effects of supervised cardio-vascular training 
program on exercise tolerance, aerobic capacity, and quality of life in patients with systemic lupus erythematosus. Arthritis and Rheumatism, 53(6), 838-844.

[23] Ramsey-Goldman, R., Schilling, E.M., Dunlop, D., Langman, C., Greenland, P., Thomas, R.J. and Chang, R.W. (2000) A pilot study on the effects of exercise in patients with systemic lupus erythematosus. Arthritis Care and Research, 13(5), 262-269.

[24] Tench, C.M., McCarthy, J., McCurdie, I., White, P.D. and D'Cruz, D.P. (2003) Fatigue in systemic lupus erythematosus: A randomized controlled trial of exercise. Rheumatology, 42(9), 1050-1054.

[25] Clarke-Jenssen, A.C., Fredriksen, P.M., Lilleby, V. and Mengshoel, A.M. (2005) Effects of supervised aerobic exercise in patients with systemic lupus erythematosus: A pilot study. Arthritis and Rheumatism, 53(2), 308-312.

[26] Oeser, A., Chung, C.P., Asanuma, Y., Avalos, I. and Stein, C.M. (2005) Obesity is an independent contributor to functional capacity and inflammation in systemic lupus erythematosus. Arthritis and Rheumatism, 52(11), 36513659.

[27] Kipen, Y., Briganti, E.M., Strauss, B.J.G., Littlejohn, G.O. and Morand, E.F. (1999) Three-year follow-up of body composition changes in pre-menopausal women with systemic lupus erythematosus. Rheumatology, 38(1), 5965.

[28] Chung, C.P., Avalos, I., Oeser, A., Gebretsadik, T., Shintani, A., Raggi, P. and Stein, C.M. (2007) High prevalence of the metabolic syndrome in patients with systemic lupus erythematosus: Association with disease characteristics and cardio-vascular risk factors. Annals of Rheumatic Disease, 66(2), 208-214.

[29] Sabio, J.M., Zamora-Pasadas, M., Jimenez-Jaimez, J., Albadalejo, F., Vargas-Hitos, J., Rodriguez del Aguila, M.D.M., Hidalgo-Tenorio, C., Gonzalez-Gay, M.A. and Alonso, J.J. (2008) Metabolic syndrome in patients with systemic lupus erythematosus from Southern Spain. $L u$ pus, 17(9), 849-859.

[30] Ekelund, U., Brage, S., Franks, P.W., Hennings, S., Emms, S. and Wareham, N.J. (2005) Physical activity energy expenditure predicts progression toward the metabolic syndrome independently of aerobic fitness in middle-aged healthy Caucasians: The Medical Research Council Ely Study. Diabetes Care, 28(5), 1195-1200. 